\title{
Key issue (Terrorism Coverage)
}

\section{AUTHOR}

Liane Rothenberger, Valerie Hase

\section{KEYWORDS}

news topics, frames, issue salience, issue ownership

\section{BRIEF DESCRIPTION}

"Key issue" describes the main issue or perspective an article focuses on when reporting on a news topic. There might be different key issues for the same topic: When reporting on terrorism, articles can for example concentrate on the incident itself, the perpetrator behind it, victims and/or political reactions to terrorism.

\section{FIELD OF APPLICATION/THEORETICAL FOUNDATION}

Key issues share similarities with other variables such as news "frames", "issue salience" or "issue ownership" that also try to identify different perspectives for the same or different news topics. Therefore, studies based on "Framing" (Entman, 1993) work with similar variables to analyze what issues journalists focus on and many studies cited here use the concept of framing to identify key issues, for example Li (2007) or Zhang \& Hellmüller (2016).

\section{REFERENCES/COMBINATION WITH OTHER METHODS OF DATA COLLECTION}

Studies for example combine content analysis and interviews with journalists to shed more light on dynamics and structures of terrorism coverage, including key issues (Larsen, 2019).

\section{EXAMPLE STUDIES}

Li (2007); Matthews (2016)

\section{INFORMATION ON LI, 2007}

Authors: $\mathrm{Li}$ (2007)

Research question: How did television outlets frame 9/11 during the first 24 hours of coverage and how did this framing change over time?

Object of analysis: News coverage by five TV outlets (ABC, CBS, NBC, CNN, and FOX news)

Time frame of analysis: 24 hours after attacks on September 92001 occurred

\section{INFO ABOUT VARIABLES}

Variable name/definition: Coverage frame: "The coverage frame is defined as the aspects of a perceived reality identified through a story that makes these aspects more salient in the news coverage" ( $\mathrm{Li}, 2007$, p. 676).

Level of analysis: News story (TV)

Variables and values: Political coverage frame, economic coverage frame, criminal coverage frame, environment coverage frame, safety coverage frame, human interest coverage frame, religion coverage frame, disaster coverage frame, other coverage frame

Reliability: Scott's pi: .8

\section{INFORMATION ON MATTHEWS, 2016}

Authors: Matthews (2016)

Research question: How did newspapers react in the immediate aftermath of the London bombings 2005?

Object of analysis: News coverage by nine UK newspapers and their Sunday equivalents (The Star, The Sun, Daily Mail, Daily Mirror, Daily Express, The Times, The Telegraph, The Guardian and The Independent)

Time frame of analysis: July 82005 to July 152005 


\section{INFO ABOUT VARIABLES}

Variable name/definition: Story themes

Level of analysis: News article

Variables and values: Reconstruction and reaction, bombers' identities, police investigation, victims/the missing, heroism and survivors, London's reaction

\section{REFERENCES}

An, Y., Mejía, N. A., Arizi, A., Villalobos, M. M, \& Rothenberger, L. (2018). Perpetrators' strategic communication: Framing and identity building on ethno-nationalist terrorists' websites. Communications, 43(2), 133-171. doi:10.1515/commun-2017-0057

Du, Y. R., \& Li, L. (2017). When press freedom meets national interest: How terrorist attacks are framed in the news in China and the US. Global Media and China, 2(3-4), 284-302. doi:10.1177/2059436418755761

Entman, R. M. (1993). Framing: Toward clarification of a fractured paradigm. Journal of Communication, 43(4), 51-58. doi:10.1111/j.1460-2466.1993.tb01304.x

Haußecker, N., \& Jirschitzka, J. (2010). Mediale Konstruktion I: Methodisches VorgehenInhaltsanalyse der Terrorberichterstattung in deutschen Fernsehnachrichten [Media construction I: Methods - content analysis of terrorism coverage in German TV news]. In W. Frindte \& N. Haußecker (Eds.), Inszenierter Terrorismus [Staged terrorism] (pp. 67-89). VS Verlag für Sozialwissenschaften.

Jirschitzka, J., Haußecker, N., \& Frindte, W. (2010). Mediale Konstruktion II: Die Konstruktion des Terrorismus im deutschen Fernsehen - Ergebnisdarstellung und Interpretation. [Media construction II: the construction of terrorism in German TV results and interpretation]. In W. Frindte \& N. Haußecker (Eds.), Inszenierter Terrorismus [Staged terrorism] (pp. 81-119). Wiesbaden: VS Verlag für Sozialwissenschaften.

Larsen, A. G. (2019). Threatening criminals and marginalized individuals: Frames and news conventions in reporting of radicalization and violent extremism. Media, War \& Conflict, 12(3), 299-316. doi:10.1177/1750635218769331

Li, X. (2007). Stages of a crisis and media frames and functions: U.S. television coverage of the $9 / 11$ incident during the first 24 hours. Journal of Broadcasting \& Electronic Media, 51(4), 670-687. doi:10.1080/08838150701626578

Li, X., \& Izard, R. (2003). 9/11 Attack coverage reveals similarities, differences. Newspaper Research Journal, 24(1), 204-219. doi:10.1177/073953290302400123

Matthews, J. (2016). Media performance in the aftermath of terror: Reporting templates, political ritual and the UK press coverage of the London Bombings, 2005. Journalism, 17(2), 173-189. doi:10.1177/1464884914554175

Zhang, X., \& Hellmüller, L. (2016). Transnational media coverage of the ISIS threat: A global perspective? International Journal of Communication, 10, 766-785. 
Table 1. Measurement of "Key Issue" in terrorism coverage.

\begin{tabular}{|c|c|c|c|c|}
\hline Author(s) & Sample & Manifestations & Reliability & Codebook \\
\hline $\begin{array}{l}\text { An et al. } \\
\text { (2018) }\end{array}$ & $\begin{array}{l}\text { Articles from } \\
\text { terrorist } \\
\text { websites }\end{array}$ & $\begin{array}{l}31 \text { different key issues, ranging } \\
\text { from terrorist attacks to their } \\
\text { political consequences }\end{array}$ & $\begin{array}{l}\text { Average Hol- } \\
\text { sti value for } \\
\text { all pairwise } \\
\text { compari- } \\
\text { sons: .66 }\end{array}$ & $\begin{array}{l}\text { Availa- } \\
\text { ble under } \\
\text { https://www. } \\
\text { hope.uzh.ch/ } \\
\text { doca/article/ } \\
\text { view/2u/1542 } \\
\text { and } \\
\text { https://www. } \\
\text { hope.uzh.ch/ } \\
\text { doca/article/ } \\
\text { view/2u/1543 }\end{array}$ \\
\hline $\begin{array}{l}\text { Du \& Li } \\
\text { (2017) }\end{array}$ & $\begin{array}{l}\text { Online news } \\
\text { articles }\end{array}$ & $\begin{array}{l}6 \text { different key issues, includ-ing } \\
\text { "description and updates of the } \\
\text { incident itself", "causes of the in- } \\
\text { cident", "conse-quences of the in- } \\
\text { cident", "conflicting viewpoint re- } \\
\text { lated to the incident", "condemn } \\
\text { the terrorist behavior and discuss } \\
\text { the punish-ment/reprisal", and } \\
\text { "back-ground/history knowledge } \\
\text { of the incident areas" }\end{array}$ & $\begin{array}{l}\text { Scott's pi for } \\
\text { all variables } \\
\text { in study: } \\
\text { between . } 798 \\
\text { and } 1\end{array}$ & $\begin{array}{l}\begin{array}{l}\text { Availa- } \\
\text { ble under } \\
\text { https://www. }\end{array} \\
\text { hope.uzh.ch/ } \\
\text { doca/article/ } \\
\text { view/2u/1544 }\end{array}$ \\
\hline $\begin{array}{l}\text { Haußecker } \\
\text { \& Jirschitz- } \\
\text { ka, 2010; } \\
\text { Jirschitzka et } \\
\text { al., } 2010\end{array}$ & $\begin{array}{l}\text { Broadcasting } \\
\text { pro-grams }\end{array}$ & $\begin{array}{l}11 \text { different key issues, rang-ing } \\
\text { from war against terror to com- } \\
\text { munication of terrorists }\end{array}$ & $\begin{array}{l}\text { Average Hol- } \\
\text { sti value for } \\
\text { all pair-wise } \\
\text { compar- } \\
\text { isons with } \\
\text { five coders } \\
\text { and one } \\
\text { main coder: } \\
.66\end{array}$ & $\begin{array}{l}\begin{array}{l}\text { Availa- } \\
\text { ble under } \\
\text { https://www. }\end{array} \\
\text { hope.uzh.ch/ } \\
\text { doca/article/ } \\
\text { view/2u/1545 } \\
\text { and } \\
\text { https://www. } \\
\text { hope.uzh.ch/ } \\
\text { doca/article/ } \\
\text { view/2u/1546 }\end{array}$ \\
\hline $\begin{array}{l}\text { Larsen } \\
(2019)\end{array}$ & $\begin{array}{l}\text { Broadcasting } \\
\text { pro-grams } \\
\text { and online } \\
\text { news articles }\end{array}$ & $\begin{array}{l}3 \text { different key issues, includ-ing } \\
\text { "threat of terrorism", "countering } \\
\text { and prevention", and "terrorism } \\
\text { as phenome-non" }\end{array}$ & $\begin{array}{l}\text { Cohen's kap- } \\
\text { pa: } .782\end{array}$ & $\begin{array}{l}\begin{array}{l}\text { Availa- } \\
\text { ble under } \\
\text { https://www. }\end{array} \\
\text { hope.uzh.ch/ } \\
\text { doca/article/ } \\
\text { view/2u/1547 } \\
\text { and } \\
\text { https://www. } \\
\text { hope.uzh.ch/ } \\
\text { doca/article/ } \\
\text { view/2u/1548 }\end{array}$ \\
\hline
\end{tabular}




\begin{tabular}{|c|c|c|c|c|}
\hline Author(s) & Sample & Manifestations & Reliability & Codebook \\
\hline Li (2007) & $\begin{array}{l}\text { Broadcasting } \\
\text { pro-grams }\end{array}$ & $\begin{array}{l}9 \text { different key issues, includ-ing } \\
\text { "political", "economic", "crimi- } \\
\text { nal", "environment", "safety", } \\
\text { "human interest", "religion", } \\
\text { "disaster", and "oth-er" coverage } \\
\text { frame }\end{array}$ & Scott's pi: .8 & Not available \\
\hline $\begin{array}{l}\text { Li \& Izard } \\
(2003)\end{array}$ & $\begin{array}{l}\text { Broadcasting } \\
\text { pro-grams } \\
\text { and news } \\
\text { articles }\end{array}$ & $\begin{array}{l}10 \text { different key issues, in-cluding } \\
\text { "business", "World Trade Center", } \\
\text { "Pentagon", "safety (concerning } \\
\text { future attacks)", "government and } \\
\text { U.S. president, "criminal activi-ty } \\
\text { and terrorism," "personal story", } \\
\text { "American public", "U.S. Arab } \\
\text { community", and "past events" }\end{array}$ & $\begin{array}{l}\text { Scott's pi for } \\
\text { all nominal } \\
\text { variables } \\
\text { in study: } \\
\text { be-tween } .78 \\
\text { and } .96\end{array}$ & Not available \\
\hline $\begin{array}{l}\text { Matthews } \\
\text { (2016) }\end{array}$ & $\begin{array}{l}\text { Newspaper } \\
\text { articles }\end{array}$ & $\begin{array}{l}6 \text { different key issues, includ-ing } \\
\text { "reconstruction and reac-tion", } \\
\text { "bombers' identities", "police in- } \\
\text { vestigation", "vic-tims/the mis- } \\
\text { sing", "heroism and survivors", } \\
\text { and "London's reaction" }\end{array}$ & Not reported & Not available \\
\hline $\begin{array}{l}\text { Zhang \& } \\
\text { Hellmüller } \\
\text { (2016) }\end{array}$ & $\begin{array}{l}\text { Online news } \\
\text { articles }\end{array}$ & $\begin{array}{l}8 \text { key issues, sorted in the over- } \\
\text { arching categories "geo-politics" } \\
\text { (consisting of "failing state", } \\
\text { "political opportunism", "strategic } \\
\text { game", "geopolitical alignment") } \\
\text { and "existential threat" (consis- } \\
\text { ting of "ISIS prowess", "human } \\
\text { rights cri-sis", "economic conse- } \\
\text { quenc-es", and "ISIS propaganda") }\end{array}$ & $\begin{array}{l}\text { Krippen- } \\
\text { dorf's alpha: } \\
.73\end{array}$ & $\begin{array}{l}\begin{array}{l}\text { Availa- } \\
\text { ble under } \\
\text { https://www. }\end{array} \\
\text { hope.uzh.ch/ } \\
\text { doca/article/ } \\
\text { view/2u/1549 } \\
\text { and } \\
\text { https://www. } \\
\text { hope.uzh.ch/ } \\
\underline{\text { doca/article/ }} \\
\text { view/2u/1550 }\end{array}$ \\
\hline
\end{tabular}

